\title{
Theory of excess noise of a quantum dot in the presence of a microwave field
}

\author{
Qing-feng Sun \\ Department of Physics, The University of Hong Kong, Pokfulam Road, Hong Kong, China \\ and State Key Laboratory for Mesoscopic Physics and Department of Physics, Peking University, Beijing 100871, China \\ Jian Wang \\ Department of Physics, The University of Hong Kong, Pokfulam Road, Hong Kong, China \\ Tsung-han Lin \\ Department of Physics, The University of Hong Kong, Pokfulam Road, Hong Kong, China \\ and State Key Laboratory for Mesoscopic Physics and Department of Physics, Peking University, Beijing 100871, China
}

(Received 24 January 2000)

\begin{abstract}
The power spectrum of excess noise of a quantum dot coupled to two leads and irradiated by a microwave field of frequency $\Omega$ is derived exactly by directly solving the Heisenberg equation. We found that for $\Gamma$ $<\hbar \Omega$, where $\Gamma$ is the linewidth of the intradot energy level, the power spectrum can be nonzero even if the averaged current is zero. This property originates from photon-assisted tunneling and is very different from the shot noise in steady state. For $\Gamma \gg \hbar \Omega$, our results are consistent with the recent experiment by Schoelkopf et al. and previous theory.
\end{abstract}

\section{INTRODUCTION}

Recently, the investigation of shot noise has become one of the most active subjects in mesoscopic physics. In contrast to equilibrium thermal noise, shot noise is a nonequilibrium, time-dependent fluctuation in current, originating from the discreteness of the charge of the carriers. ${ }^{1,2}$ Since the shot noise reflects the temporal correlations of the current fluctuations, it can provide more information on the kinetics of the electrons than the conductance and the current-voltage characteristics, which are related only to the time-averaged current. ${ }^{3,4}$ Up to now, most of the studies on shot noise in mesoscopic systems have concentrated on steady-state transport phenomena. The main issue is to study the suppression or enhancement of the power spectrum of the shot noise, either by the Pauli exclusion principle or by Coulomb interactions between electrons. ${ }^{5}$ Another interesting problem is how to probe the high frequency behavior of the power spectrum of shot noise. Recently, Aguado and Kouwenhoven proposed a measurement setup for detecting quantum noise over a wider frequency range using inelastic transitions in a tunable two-level systems as a detector. ${ }^{6}$ The systems studied include quantum point contacts, double-barrier junctions, chaotic cavities, disordered conductors, normal-metalsuperconductor hybrid structures, quantum Hall effect systems, ferromagnetic single-electron tunneling devices, etc. (see the references in Refs. 1 and 3).

Shot noise involving time-dependent transport phenomena has received less attention. Several years ago, Lesovik and Levitov ${ }^{7}$ proposed a gedanken experiment with a conductor driven by $\mathrm{dc}$ and ac external fields in which they studied the shot noise affected by ac excitation, and found that it had a quite different behavior from the current, due to two-particle interference. ${ }^{8}$ Very recently, Schoelkopf et al. ${ }^{9}$ investigated the shot noise in time-dependent transport of a diffusive metallic conductor irradiated by a monochromatic microwave (MW) field. They observed the "photonassisted" shot noise of a phase-coherent wire. The observed power spectrum exhibits frequency-dependent features having all the signatures of photon-assisted tunneling (PAT), while the conductance is completely unaffected by the MW field because of the linearity of the system. ${ }^{9}$

Various effects of PAT on transport properties, such as the linear conductance and the $I-V$ characteristics, have been studied extensively for a long time. ${ }^{10}$ In the last decade, PAT has been investigated in a variety of systems, including quantum dots ${ }^{11}$ double quantum dots, ${ }^{12}$ semiconductor superlattices, ${ }^{13}$ and mesoscopic superconductor-normal hybrid systems. ${ }^{14}$ The essential effect of PAT on transport properties is that the electrons tunneling through the system can exchange energy with MW fields, opening new inelastic tunneling channels and introducing many effects such as the sideband effect, the turnstile effect, photon-electron pumping, etc. ${ }^{15}$

In this paper, we study the shot noise of a quantum dot system coupled to two leads irradiated by MW fields. Two different frequency regimes have been studied in detail. For $\Gamma<\hbar \Omega$, we find the following. (1) The power spectrum of the shot noise can be nonzero even if the averaged current is zero, which originates from the PAT. This is very different from the shot noise in steady state where the shot noise must be zero if the current vanishes. ${ }^{1}$ (2) $d S_{0} / d V_{\mathrm{dc}}^{L}$ vs $\epsilon_{0}$ is asymmetric for the $n$-PAT and $-n$-PAT processes ( $n$ is an integer), quite different from the behavior of the conductance, which is symmetric with $\epsilon_{0}$. For $\Gamma \gg \hbar \Omega$, our results are very consistent with the recent experiment by Schoelkopf et al. ${ }^{9}$ and the previous theory by Lesovik and Levitov ${ }^{7}$ for a single-channel coherent conductor with a time-dependent potential.

\section{MODEL AND FORMULATION}

In order to study the important physics of shot noise affected by MW fields, we shall focus on transport in the 
neighborhood of a single Coulomb oscillation peak. Therefore we can simply assume that the dot has only one single state and the Coulomb interaction can also be neglected. Then the system is described by the following Hamiltonian:

$$
\begin{aligned}
H= & \sum_{k, \beta \in L, R} \epsilon_{k \beta}(t) a_{k \beta}^{\dagger} a_{k \beta} \\
& +\left(\sum_{k, \beta \in L, R} v_{k \beta} a_{k \beta}^{\dagger} c+\text { H.c. }\right)+\epsilon_{0} c^{\dagger} c,
\end{aligned}
$$

where $a_{k L}^{\dagger}\left(a_{k L}\right), a_{k R}^{\dagger}\left(a_{k R}\right)$, and $c^{\dagger}(c)$ are creation (annihilation) operators in the left lead, the right lead, and the dot, respectively. The term in parentheses denotes the tunneling part which is time independent. Here the dot energy $\epsilon_{0}$ is controlled by the gate voltage $v_{g}: \epsilon_{0}\left(v_{g}\right)=\epsilon_{0}^{0}-e v_{g} .{ }^{16} \mathrm{We}$ assume that the MW fields are applied only on the two leads, not on the dot. Under the adiabatic approximation, the MW field causes only a rigid shift of the single-electron energy $\epsilon_{k \beta}(\beta=L, R), \epsilon_{k \beta}(t)=\epsilon_{k}-e V_{d c}^{\beta}-e V_{a c}^{\beta} \cos \Omega t$, but does not change the distributions of the electrons in the left and the right leads. ${ }^{17}$

The current operator $\hat{j}_{L}(t)$ of the electrons flowing from the left lead into the quantum dot can be calculated from the evolution of the total number operator of the electrons in the left leads, $\hat{j}_{L}(t)=-(d / d t) \sum_{k} a_{k L}^{\dagger}(t) a_{k L}(t)$ (in units of $\hbar=e$ $=1)$. Then we deduce the operators $a_{k \beta}(t)$ and $c(t)$ by directly solving the Heisenberg equations. First we assume that the tunneling term between the dot and two leads is turned on at time $t_{0}$, i.e., for $t<t_{0}$, the three subsystems (the left lead, the dot, and the right lead) are in local equilibrium states, which gives the initial conditions for the Heisenberg equations. So for $t>t_{0}$ from the Heisenberg equations one has

$$
\begin{gathered}
i \frac{\partial}{\partial t} c(t)=\epsilon_{0} c(t)+\sum_{k, \beta \in L, R} v_{k \beta}^{*} a_{k \beta}(t), \\
i \frac{\partial}{\partial t} a_{k \beta}(t)=\epsilon_{k \beta}(t) a_{k \beta}(t)+v_{k \beta} c(t) .
\end{gathered}
$$

Introducing the linewidth function of the intradot state $\epsilon_{0}$, $\Gamma_{\beta}(\epsilon) \equiv 2 \pi \rho_{\beta}(\epsilon)\left|v_{\beta}(\epsilon)\right|^{2}(\beta=L, R)$, in which $\rho_{\beta}(\epsilon)$ is the density of states in lead $\beta$ and $v_{\beta}\left(\epsilon_{k}\right)=v_{k \beta}$; and taking the wide-bandwidth approximation, ${ }^{14}$ under which $\Gamma_{\beta}$ is independent of energy, the coupled differential equations Eqs. (2) and (3) [with the initial condition $\left.a_{k \beta}(t)\right|_{t=t_{0}}=a_{k \beta}\left(t_{0}\right)$ and $\left.c(t)\right|_{t=t_{0}}=c\left(t_{0}\right)$ ], can be solved exactly:

$$
\begin{aligned}
c(t)= & e^{-\left(i \epsilon_{0}+\Gamma / 2\right)\left(t-t_{0}\right)} c\left(t_{0}\right)-i e^{-\left(i \epsilon_{0}+\Gamma / 2\right) t} \\
& \times \int_{t_{0}}^{t} d t_{1}\left[A_{L}\left(t_{1}\right)+A_{R}\left(t_{1}\right)\right], \\
a_{k \beta}(t)= & \exp \left(-i \int_{t_{0}}^{t} \epsilon_{k \beta}(\tau) d \tau\right)\left[a_{k \beta}\left(t_{0}\right)-i \int_{t_{0}}^{t} d t_{1}\right. \\
& \left.\times \exp \left(i \int_{t_{0}}^{t_{1}} \epsilon_{k \beta}(\tau) d \tau\right) v_{k \beta} c\left(t_{1}\right)\right],
\end{aligned}
$$

where $\Gamma \equiv \Gamma_{L}+\Gamma_{R}$, and $A_{\beta}(t)(\beta=L, R)$ is defined as

$$
A_{\beta}(t)=e^{\left(i \epsilon_{0}+\Gamma / 2\right) t} \sum_{k} v_{k \beta}^{*} \exp \left(-i \int_{t_{0}}^{t} \epsilon_{k \beta}(\tau) d \tau\right) a_{k \beta}\left(t_{0}\right) .
$$

Then we let $t_{0} \rightarrow-\infty$, noting that the terms including $c\left(t_{0}\right)$ in Eqs. (4) and (5) approach zero, which means that the system's behavior at time $t\left(t>t_{0}\right)$ does not correlate with the initial $\left(t_{0}=-\infty\right)$ occupation number on the dot. Substituting Eq. (5) into the current operator, one has

$$
\hat{j}_{L}(t)=i\left[e^{\left(i \epsilon_{0}-\Gamma / 2\right) t} A_{L}^{\dagger}(t) c(t)-\text { H.c. }\right]-e \Gamma_{L} c^{\dagger} c(t) .
$$

Let $I_{L}(t)=\left\langle\hat{j}_{L}(t)\right\rangle$, where $\langle\cdots\rangle$ denotes the ensemble average for the state at $t_{0}=-\infty$. Then one can obtain the timedependent and time-averaged currents immediately; they are the same as those obtained by the nonequilibrium Green function method. ${ }^{18}$ Now we proceed to calculate the noise, which is generally defined as ${ }^{19} S(t) \equiv\left\langle\Delta \hat{j}_{L}\left(t_{1}\right) \Delta \hat{j}_{L}\left(t_{1}+t\right)\right.$ $\left.+\Delta \hat{j}_{L}\left(t_{1}+t\right) \Delta \hat{j}_{L}\left(t_{1}\right)\right\rangle_{t_{1}}$, where $\Delta \hat{j}_{L}(t) \equiv \hat{j}_{L}(t)-\left\langle\hat{j}_{L}(t)\right\rangle$ and the subscript $t_{1}$ stands for the time average over $t_{1}$. Substituting the current operator $\hat{j}(t)$ into this definition and taking the Fourier transformation, the spectral density of the noise can be obtained exactly. Here we show only the zerofrequency spectral density $S_{0}$ :

$$
\begin{aligned}
S_{0}= & 2 \sum_{n, m, n_{1}, m_{1}} \int \frac{d \epsilon}{2 \pi} \frac{\delta_{n+n_{1}, m+m_{1}} \Gamma_{L} \Gamma_{R}}{\left(E_{m}^{2}+\Gamma^{2} / 4\right)\left(E_{n}^{2}+\Gamma^{2} / 4\right)} \\
& \times \sum_{\beta \in L, R} J_{n}\left(\alpha_{\beta}\right) J_{m}\left(\alpha_{\beta}\right)\left\{J_{n_{1}}\left(\alpha_{\beta}\right) J_{m_{1}}\left(\alpha_{\beta}\right) \Gamma_{L} \Gamma_{R} f_{\beta}(\epsilon)\right. \\
& \times\left[1-f_{\beta}\left(\epsilon^{\prime}\right)\right]+J_{n_{1}}\left(\alpha_{\bar{\beta}}\right) J_{m_{1}}\left(\alpha_{\bar{\beta}}\right) f_{\beta}(\epsilon)\left[1-f_{\bar{\beta}}\left(\epsilon^{\prime}\right)\right] \\
& \left.\times\left[E_{m} E_{n}+\Gamma^{2} / 4-\Gamma_{L} \Gamma_{R}\right]\right\},
\end{aligned}
$$

in $\quad$ which $\quad E_{m} \equiv \epsilon-\epsilon_{0}+m \Omega, \quad \epsilon^{\prime} \equiv \epsilon+m \Omega-n_{1} \Omega, \quad \alpha_{\beta}$ $=e V_{\mathrm{ac}}^{\beta} / \Omega(\beta=L, R)$, and $\bar{\beta}=R$ if $\beta=L$, otherwise $\bar{\beta}=L$. Equation (8) represents the total noise spectral density, including the thermal noise and the shot noise. Because of the opening of multiple inelastic tunneling channels, the expression for the noise becomes more complicated. Without the MW fields, $S_{0}$ reduces to the well-known steady-state formula,

$$
\begin{aligned}
S_{0}= & 2 \int \frac{d \epsilon}{2 \pi} \sum_{\beta=L, R}\left\{T^{2} f_{\beta}(\epsilon)\left[1-f_{\beta}(\epsilon)\right]\right. \\
& \left.+T(1-T) f_{\beta}(\epsilon)\left[1-f_{\bar{\beta}}(\epsilon)\right]\right\},
\end{aligned}
$$

in which $T(\epsilon)=\Gamma_{L} \Gamma_{R} /\left(E_{0}^{2}+\Gamma^{2} / 4\right)$ is the Breit-Wigner transmission probability. Note that Eq. (9) consists of the thermal noise which vanishes at zero temperature and the shot noise which exists only for $\langle I\rangle \neq 0$. If $\mathcal{T}=0$ and $\langle I\rangle$ $=0$, the total noise is zero. However, in the presence of MW fields, since to the electrons tunneling through the dot can absorb or emit photons, both thermal noise and shot noise 


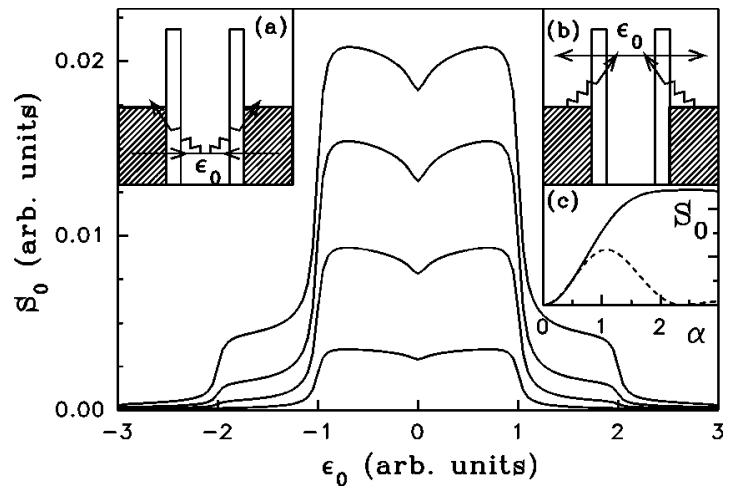

FIG. 1. $S_{0}$ vs $\epsilon_{0}$ at zero bias $\left(V_{\mathrm{dc}}^{L}=V_{\mathrm{dc}}^{R}=0\right)$; where $\Gamma_{L}=\Gamma_{R}$ $=0.05, \mathcal{T}=0$, and $\Omega=1$. Different curves correspond to $\alpha_{L}=\alpha_{R}$ $=0.4,0.7,1.0$, and 1.4 from bottom to top. Insets (a) and (b) are schematic diagrams showing the PAT processes which can cause shot noise but not the averaged current. (a) $\epsilon_{0}=-\Omega$; (b) $\epsilon_{0}=\Omega$. The inset (c) shows $S_{0}$ vs $\alpha$ at $\epsilon_{0}=0.5$ (solid curve), and the dashed curve is $\Gamma J_{0}^{2}(\alpha) J_{1}^{2}(\alpha)$ vs $\alpha$ for comparison.

will change significantly. The noise becomes nonzero even if $\mathcal{T}=0$ and $\langle I\rangle=0$. In the following, we study two different regimes: $\Gamma<\Omega$ and $\Gamma \gg \Omega$.

\section{THE CASE WITH $\Gamma<\Omega$}

This is the typical parameter region for quantum dot systems. ${ }^{11,12}$ For this case, the effects of PAT on the averaged current or the conductance have been extensively

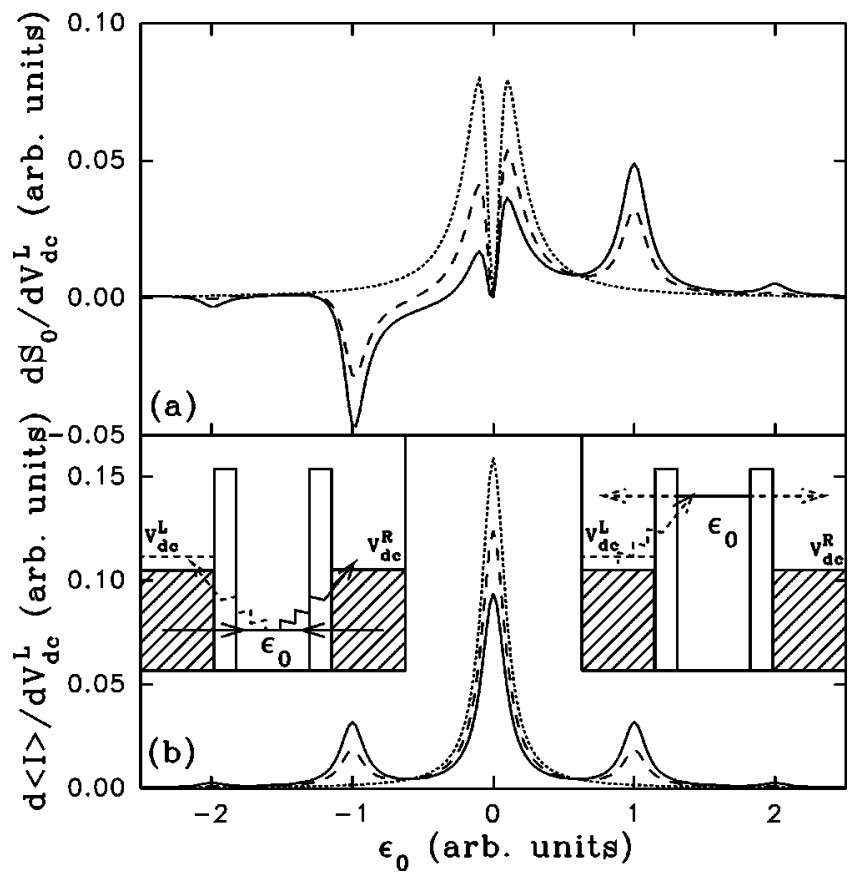

FIG. 2. (a) $d S_{0} / d V_{\mathrm{dc}}^{L}$ vs $\epsilon_{0}$. (b) $d\langle I\rangle / d V_{\mathrm{dc}}^{L}$ vs $\epsilon_{0}$. The parameters are $\Gamma_{L}=\Gamma_{R}=0.1, \mathcal{T}=0, \Omega=1$, and $V_{\mathrm{dc}}^{L}=V_{\mathrm{dc}}^{R}=0$. Different curves correspond to $\alpha_{L}=\alpha_{R}=0$ (dotted curve), 0.7 (dashed curve), and 1.0 (solid curve). The left $\left(\epsilon_{0}=-\Omega\right)$ and right $\left(\epsilon_{0}=\Omega\right)$ insets in (b) are schematic diagrams showing the variation of PAT processes when $V_{\mathrm{dc}}^{L}$ changes slightly. The change of the net current is positive in both insets, but the change of the noise is negative in the left and positive in the right inset.

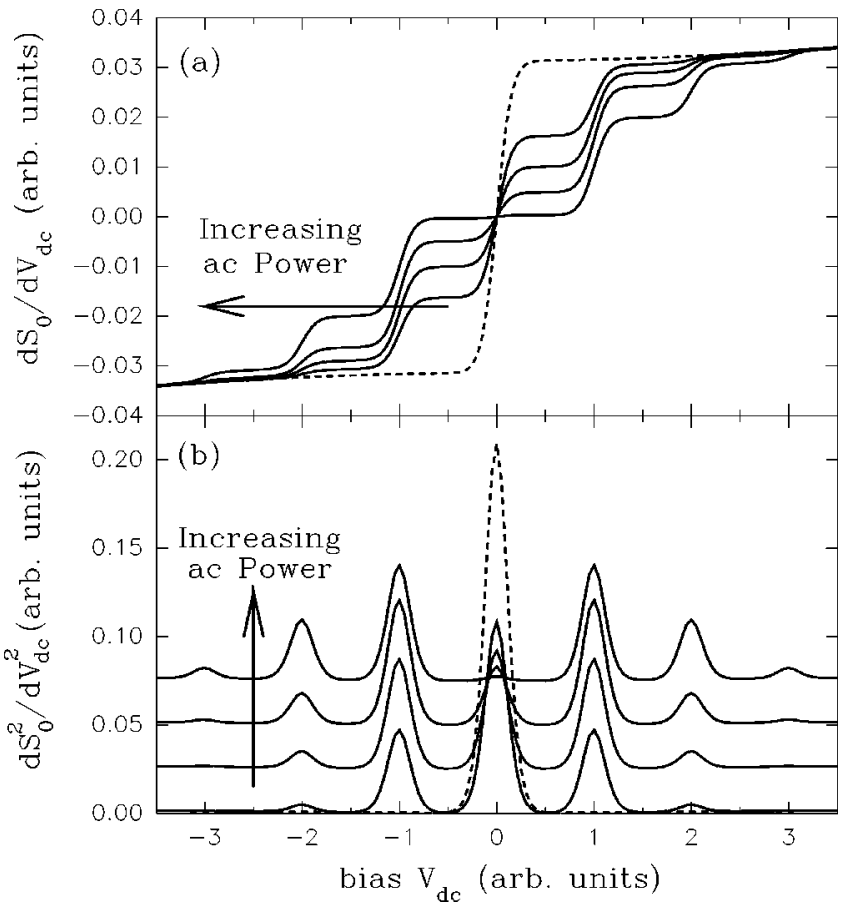

FIG. 3. (a) $d S_{0} / d V_{\mathrm{dc}}$ vs $V_{\mathrm{dc}}$; (b) $d S_{0}^{2} / d V_{\mathrm{dc}}^{2}$ vs $V_{\mathrm{dc}}$. The parameters are $\Gamma_{L}=40, \Gamma_{R}=20, \epsilon_{0}=0, \Omega=1$, and $\mathcal{T}=0.05$. Different curves correspond to $\alpha_{L}=-\alpha_{R}=0$ (dashed curve), 1.1, 1.4, 1.7, and 2.2, respectively. Different solid curves in (b) have been offset by 0.025 along the vertical axis for clarity.

investigated. ${ }^{11}$ In the following, we set temperature $\mathcal{T}=0$ and focus on the properties of the excess noise in the presence of photon-assisted processes. Figure 1 presents $S_{0}$ vs $\epsilon_{0}$ at zero dc bias and for symmetrical MW fields (i.e., $\alpha_{L}=\alpha_{R}=\alpha$ ). Note that for this case (1) the averaged current $I$ is always zero, no matter whether the MW fields exist or not; (2) without the MW fields, one has $S_{0}=0$ since the shot noise is related to the current in the steady state. However, in the presence of the MW fields, the shot noise will be nonzero. This is due to the fact that the electrons tunneling through the barriers can absorb or emit photons, opening extra inelastic channels. Around $\epsilon_{0}=n \Omega(n= \pm 1, \pm 2, \ldots)$, the shot noise changes significantly, within the energy range of $\Gamma$. This can be understood as follows. While $\epsilon_{0}=-\Omega$ (or $0>\epsilon_{0}>-\Omega$ ), electrons of the two leads can first tunnel into the intradot level $\epsilon_{0}$, and then by absorbing a photon tunnel out of the dot through the left or the right barrier to the left or right lead [see inset (a) in Fig. 1]. Note that this process does not give rise to any net averaged current, but it can cause shot noise. Therefore, the shot noise increases dramatically when $\epsilon_{0}$ passes through $-\Omega$. Similarly, the shot noise decreases when $\epsilon_{0}$ passes through $\Omega$ [see inset (b) of Fig. 1]. The inset (c) of Fig. 1 shows $S_{0}$ vs $\alpha$ at $\epsilon_{0}=0.5$ (solid curve) and the function $\Gamma J_{0}^{2}(\alpha) J_{1}^{2}(\alpha)$ (dashed curve), which is the noise due to the process depicted in inset (a) of Fig. 1. The two curves are almost identical for small $\alpha$ indicating that the shot noise really originates from the PAT processes described above. For large $\alpha$, multiple photon processes become more and more important, causing the difference between the two curves. In Fig. 1 one sees that a small dip appears when $\epsilon_{0}$ approaches zero. For example, as $\epsilon_{0}$ approaches zero from positive values, the dot state will gradu- 
ally be occupied by electrons because of the finite width of the resonant state (if $\epsilon_{0}=0$, the occupation number in the state just equals $1 / 2$ ), so the PAT processes mentioned above [inset (b) of Fig. 1] will be slightly blocked by the Pauli exclusion principle, leading to a slight decrease of the shot noise.

Figure 2(a) shows $d S_{0} / d V_{\mathrm{dc}}^{L}$ vs $\epsilon_{0}$, while the conductance $d\langle I\rangle / d V_{\mathrm{dc}}^{L}$ is also shown in Fig. 2(b) for comparison (for the behavior of $d\langle I\rangle / d V_{\mathrm{dc}}^{L}$, see Refs. 11 and 18). The dotted curve in Fig. 2(a) denotes the steady-state case, showing two maxima at $\epsilon_{0}= \pm \Gamma / 2$, corresponding to the transmission probability $T=1 / 2$; while the dashed and solid curves represent the results with PAT. In addition to the positive peaks emerging at $\epsilon_{0}=n \Omega(n=1,2, \ldots)$, there are also negative peaks at $\epsilon_{0}=-n \Omega$. Notice that this property is very different from the behavior of the conductance where all $n$-PAT processes give positive peaks. The heights of the peaks vary nonmonotonically with the power of the MW field. This can be understood from the insets of Fig. 2 as follows. (1) If $\epsilon_{0}$ is slightly larger than $\Omega$, the electrons at the Fermi level of the two leads cannot jump into the intradot level $\epsilon_{0}$, even by absorbing a photon. However, when $V_{\mathrm{dc}}^{L}$ increases slightly, the process become possible [as shown by the dashed arrows in the right inset of Fig. 2(b)], leading to an increase of the shot noise. Therefore, a positive peak emerges at $\Omega$. (2) On the contrary, when $\epsilon_{0}=-\Omega$, the electrons at the level $\epsilon_{0}$ can jump to a state above the Fermi level of the left lead. However, if $V_{\mathrm{dc}}^{L}$ increases slightly [see the left inset in Fig. 2(b)], this process will be prohibited by the Pauli exclusion principle, leading to a decrease of the shot noise. So a negative peak emerges at $\epsilon=-\Omega$. Note that the averaged current also changes in the above two processes, but the changes are always positive.

\section{THE CASE WITH $\Gamma \gg \Omega$}

Let $V_{\mathrm{dc}}\left(V_{\mathrm{dc}}=V_{\mathrm{dc}}^{L}-V_{\mathrm{dc}}^{R}\right)$ vary in a range of a few $\Omega$ and set $\epsilon_{0}$ to be zero, so one also has $\Gamma \gg V_{\mathrm{dc}}$. By setting $\alpha$ $\equiv \alpha_{L}-\alpha_{R}$, our noise formula, Eq. (8), will approximately reduce to the result obtained by Lesovik and Levitov [see Eq. (1) in Ref. 9). Figure 3 shows $d S_{0} / d V_{\mathrm{dc}}$ and $d^{2} S_{0} / d V_{\mathrm{dc}}^{2}$ vs
$V_{\mathrm{dc}}$. Here we take $\mathcal{T} \neq 0$ but very small and $V_{\mathrm{ac}}^{L}=-V_{\mathrm{ac}}^{R}$ (i.e., $\alpha_{L}=-\alpha_{R}$, which is the situation in the experiment by Schoelkopf et al.). In Fig. 3(a) one can clearly see that $d S_{0} / d V_{\mathrm{dc}}$ vs $V_{\mathrm{dc}}$ exhibits a series of steps, with a step width of $\Omega$ and heights proportional to $J_{n}^{2}(\alpha)(n=0, \pm 1, \pm 2, \ldots)$. Correspondingly, $d^{2} S_{0} / d V_{\mathrm{dc}}^{2}$ vs $V_{\mathrm{dc}}$ exhibits a series of peaks with the peak spacing of $\Omega$, heights related to $J_{n}^{2}(\alpha)$, and the half-width of the peak determined by temperature $\mathcal{T}$. It should be mentioned that the averaged current $I$ is approximately equal to $4 e \Gamma_{L} \Gamma_{R} V_{\mathrm{dc}} / 2 \pi \Gamma$, independent of the MW field. These results are very consistent with the experiment by Schoelkopf et al. ${ }^{9}$ and the previous theory by Lesovik and Levitov. ${ }^{7}$ This is because, in this case, the quantum dot is strongly coupled to the two leads, so the dot in the resonant tunneling condition is approximately equivalent to a singleconducting-channel conductor, similar to the system studied in the experiment by Schoelkopf et al. ${ }^{9}$

It should also be pointed out that, for $\Gamma \gg \Omega, S_{0}$ will still be nonzero even if $\langle I\rangle=0$ and $\mathcal{T}=0$.

\section{SUMMARY}

In this paper we investigated the excess noise in a quantum dot system in the presence of photon-assisted processes. For the case of $\Gamma<\Omega$, we found that the excess power spectrum of the shot noise can be nonzero even if the averaged current is zero; and $d S_{0} / d V_{\mathrm{dc}}^{L}$ vs $\epsilon_{0}$ is asymmetric for the $n$-PAT and $-n$-PAT processes, quite different from the behavior of the conductance, which is symmetric with $\epsilon_{0}$. This result has not been reported before to our knowledge and deserves further experimental tests. For the opposite case of $\Gamma \gg \Omega$, our results are very consistent with the recent experiment by Schoelkopf at al. and the previous theory by Lesovik and Levitov.

\section{ACKNOWLEDGMENTS}

We gratefully acknowledge financial support by a RGC grant from the SAR Government of Hong Kong under Grant No. HKU 7115/98P, a research grant from the Chinese National Natural Science Foundation, and the Doctoral Program Foundation of the Institution of Higher Education.
${ }^{1}$ For a review, see M.J.M. de Jong and C.W.J. Beenakker, in $\mathrm{Me}$ soscopic Electron Transport, edited by L.L. Sohn, L.P. Kouwenhoven, and G. Schön (Kluwer, Dordrecht, 1997).

${ }^{2}$ M. Büttiker, Phys. Rev. Lett. 65, 2901 (1990); Phys. Rev. B 46, 12485 (1992).

${ }^{3}$ R. Landauer, Nature (London) 392, 658 (1998).

${ }^{4}$ T. Gramespacher and M. Büttiker, Phys. Rev. Lett. 81, 2763 (1998).

${ }^{5}$ G. Iannaccone et al., Phys. Rev. Lett. 80, 1054 (1998); Y.D. Wei, B.G. Wang, J. Wang, and H. Guo, Phys. Rev. B 60, 16900 (1999).

${ }^{6}$ R. Aguado and L.P. Kouwenhoven, cond-mat/9905290 (unpublished).

${ }^{7}$ G.B. Lesovik and L.S. Levitov, Phys. Rev. Lett. 72, 538 (1994).

${ }^{8}$ M. Büttiker, Phys. Rev. Lett. 68, 843 (1992).
${ }^{9}$ R.J. Schoelkopf et al., Phys. Rev. Lett. 80, 2437 (1998).

${ }^{10}$ P.K. Tien and J.P. Gordon, Phys. Rev. 129, 647 (1963).

${ }^{11}$ L.P. Kouwenhoven et al., Phys. Rev. Lett. 73, 3443 (1994); T.H. Oosterkamp et al., ibid. 78, 1536 (1997).

${ }^{12}$ C.A. Stafford and N.S. Wingreen, Phys. Rev. Lett. 76, 1916 (1996); T.H. Oosterkamp et al., Nature (London) 395, 873 (1998).

${ }^{13}$ B.J. Keay et al., Phys. Rev. Lett. 75, 4098 (1995); 75, 4102 (1995).

${ }^{14}$ Q.-F. Sun, J. Wang, and T.-H. Lin, Phys. Rev. B 59, 13126 (1999).

${ }^{15}$ L. P. Kouwenhoven et al., in Mesoscopic Electron Transport (Ref. 1), and references therein.

${ }^{16}$ Because of the gate voltage, the gauge invariant condition is satisfied. For more discussion of gauge invariance in PAT, see 
M.H. Pedersen and M. Büttiker, Phys. Rev. B 58, 12993 (1998), where the general noise spectrum has been derived using scattering matrix theory.

${ }^{17}$ N.S. Wingreen, A.-P. Jauho, and Y. Meir, Phys. Rev. B 48, 8487
(1993); A.-P. Jauho, N.S. Wingreen, and Y. Meir, ibid. 50, 5528 (1994).

${ }^{18}$ Q.-F. Sun and T.-H. Lin, Phys. Rev. B 56, 3591 (1997).

${ }^{19}$ G.-H. Ding and T.-K. Ng, Phys. Rev. B 56, R15 521 (1997). 